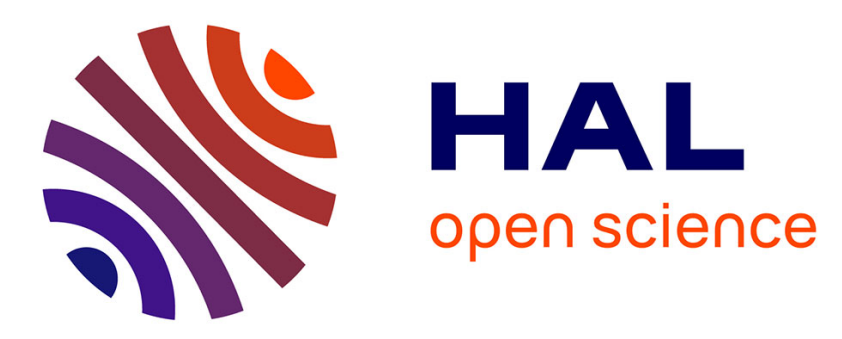

\title{
Synchronization of Perturbed Linear Systems with Data-Rate Constraints
}

Quentin Voortman, Denis Efimov, Alexander Yu Pogromsky, Jean-Pierre Richard, Henk Nijmeijer

\section{- To cite this version:}

Quentin Voortman, Denis Efimov, Alexander Yu Pogromsky, Jean-Pierre Richard, Henk Nijmeijer. Synchronization of Perturbed Linear Systems with Data-Rate Constraints. CDC 2020 - 59th IEEE Conference on Decision and Control, Dec 2020, Jeju Island / Virtual, South Korea. hal-02942416

\author{
HAL Id: hal-02942416 \\ https://hal.inria.fr/hal-02942416
}

Submitted on 17 Sep 2020

HAL is a multi-disciplinary open access archive for the deposit and dissemination of scientific research documents, whether they are published or not. The documents may come from teaching and research institutions in France or abroad, or from public or private research centers.
L'archive ouverte pluridisciplinaire HAL, est destinée au dépôt et à la diffusion de documents scientifiques de niveau recherche, publiés ou non, émanant des établissements d'enseignement et de recherche français ou étrangers, des laboratoires publics ou privés. 


\title{
Synchronization of Perturbed Linear Systems with Data-Rate Constraints
}

\author{
Quentin Voortman, Denis Efimov, Alexander Yu. Pogromsky, Jean-Pierre Richard and Henk Nijmeijer
}

\begin{abstract}
In this paper, we consider the synchronization of two linear systems that are subject to unknown perturbations. One of the systems is driven by a reference signal, which is unknown to the second system. Both systems can only use a one-way communication channel to exchange information. The channel is subject to data-rate constraints. The messages are generated by a smart sensor that measures the state and is capable of performing some computations. The messages are received by a controller which interprets them to apply an appropriate control input to the system. The objective is to design a sensor/controller pair, which we will refer to as a communication protocol, such that the distance between the states of both systems is bounded, and as small as possible communication rate is necessary. In this paper, a communication protocol that achieves this objective is presented together with the minimum channel rate to implement it. Simulations of the communication protocol are provided to support the theoretical work.
\end{abstract}

\section{INTRODUCTION}

The widespread usage of wireless technologies in industry has created a new area in the field of dynamics and control that takes into account the data-rate constrained channels. All problems studied in this sub-field share common features: one or several dynamical systems, and possibly their sensors, controllers, actuators, are placed at locations remote from one another. The different devices are connected via communication channels which can only transmit limited amounts of data per unit of time, hence the name data-rate constrained communication channels. The many examples of such applications include remote sensors that communicate via Wi-Fi, microelectromechanical systems (where the constraints are due to the size of the components), platoons of connected vehicles, formation control for drones, etc... The fact that these data-rate constrained channels are connected to dynamical systems implies that it is necessary to design specific strategies for that situation, as opposed to simply relying on classical signal theory or control and estimation theory separately. For most problems, the need for persistent communication is due to one or several sources

Quentin Voortman, Alexander Yu. Pogromsky and Henk Nijmeijer are with the Department of Mechanical Engineering, Eindhoven Univ. of Technology, Eindhoven, The Netherlands. Alexander Yu. Pogromsky is also with the Department of Control Systems and Robotics, ITMO, St. Petersburg, Russia. Quentin Voortman, together with Denis Efimov and Jean-Pierre Richard are with Inria, Univ. of Lille, CNRS, UMR 9189-CRIStAL, Centrale Lille F-59000 Lille, France $\{q . j . t . v o o r t m a n @ t u e . n l\}$.

This paper was elaborated in the UCoCoS project which has received funding from the European Union's Horizon 2020 research and innovation programme under the Marie Skłodowska-Curie grant agreement No 675080. It was also supported by the Government of Russian Federation (Grant 08-08) and by the Ministry of Science and Higher Education of Russian Federation, passport of goszadanie no. 2019-0898 of uncertainty (such as uncertainty in initial conditions, parametric uncertainties, perturbations, noise, etc).

One of the earliest works of this sub-field of dynamical systems and data-rate constrained communication channels is [14], where the state estimation was considered. Another early paper is [4], which provided a solution for the stabilization of linear systems via data-rate constrained communication channels. After these two early papers, many other works considered the case of control and estimation of linear systems. Broad overviews of these results can be found in [10], [2] and [1].

Some later papers focused not on the problem of estimation and control, but of synchronization of two or more dynamical systems. Two of the earliest results are [6] and [5], where the problem of master/slave synchronization of two nonlinear systems was considered. Many other papers have focused on this problem, both for linear and nonlinear systems. Such papers include [8], which considered the problem of average consensus in networks of linear systems with fixed topologies. In [9] an extension of the previous results with time-varying network topologies was presented. In [7] an event-triggered approach was developed for the average consensus of linear systems with data-rate constraints. Some papers have dealt with the problem of consensus of nonlinear systems with data-rate constraints such as [3] and [12].

In this paper, a communication scheme is developed for the synchronization of two linear systems with perturbations and bounded measurement errors, where one of the systems is driven by a reference signal that the second system doesn't have. The systems are connected via a one-way data-rate constrained communication channel, which they have to use to exchange the information necessary for their synchronization. The main contribution of this paper is a new communication scheme, which allows us to maintain a bounded distance between the states of both systems, whilst requiring a low channel rate to function. By using an event-triggered strategy, which is the novelty of the result, the communication scheme is designed such that it can sometimes use much less than the theoretical minimum required channel rate.

\section{Problem Statement}

We consider two linear discrete-time dynamical systems. The first system is

$$
\begin{aligned}
x_{1}[k+1] & =A x_{1}[k]+B r[k]+d_{1}[k], \\
y_{1}[k] & =x_{1}[k]+w_{1}[k], \\
x_{1}[0] & =x_{10},
\end{aligned}
$$




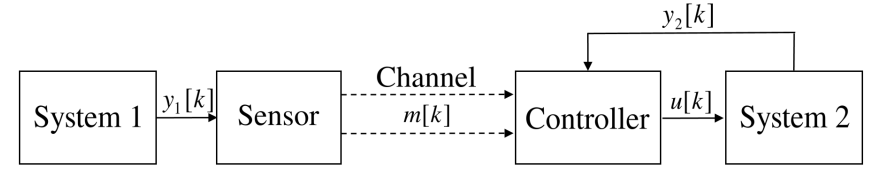

Fig. 1. Structure of the setup.

and the second system is

$$
\begin{aligned}
x_{2}[k+1] & =A x_{2}[k]+B u[k]+d_{2}[k], \\
y_{2}[k] & =x_{2}[k]+w_{2}[k], \\
x_{2}[0] & =x_{20},
\end{aligned}
$$

for $i \in\{1,2\}$, with $A \in \mathbb{R}^{n_{x} \times n_{x}}, x_{i}[k] \in \mathbb{R}^{n_{x}}$ are the states of the system, $B \in \mathbb{R}^{n_{x} \times n_{r}}, r[k] \in \mathbb{R}^{n_{r}}$ is an external reference signal, $u[k] \in \mathbb{R}^{n_{r}}$ is the input of the second system, $d_{i}[k] \in \mathbb{R}^{n_{x}}$ are the state perturbations, $y_{i}[k] \in \mathbb{R}^{n_{x}}$ are the measured outputs, and $w_{i}[k] \in \mathbb{R}^{n_{x}}$ are the measurement noises. We introduce the following hypothesis about the state perturbations and the measurement noises.

Assumption 1: The state perturbations and measurement noises are unknown signals verifying $\left\|d_{i}[k]\right\|_{2} \leq \delta$, $\left\|w_{i}[k]\right\|_{2} \leq \omega$, for $i \in\{1,2\}, \forall k \geq 0$, where $\delta$ and $\omega$ are known constants.

We make the following assumptions about the external reference signal $r[k]$.

Assumption 2: The signal $r[k] \in R$ for all $k \geq 0$, where $R \subset \mathbb{R}^{n_{r}}$ is a known bounded set of control inputs. The signal $r[k]$ is not known in advance by the communication peer associated with system (1), but measured in real time, without any measurement error.

The previous assumption is motivated by the fact that in any practical examples, the possible control inputs are always bounded by physical constraints. We will assume that at the start of the communication protocol, the state of both systems are within a certain distance $\varepsilon$ of each other, where $\varepsilon>0$ is a parameter to be chosen as a part of the communication protocol.

Assumption 3: The following holds for the initial states $x_{10}$ and $x_{20}:\left\|x_{10}-x_{20}\right\|_{2} \leq \varepsilon$.

Remark 1: We do not consider the case where the output is some linear combination of the states, possibly of lower dimension than $n_{x}$ (i.e. $y_{i}[k]=C x_{i}[k]+\bar{w}_{i}[k], C \neq I_{n_{x}}$, where $I_{n}$ is the $n \times n$ identity matrix) since it was proven in [11] that this situation is equivalent to $y_{i}[k]=x_{i}[k]+w_{i}[k]$ with a larger $\omega$.

Both systems are placed at locations remote from one another and can only communicate via a data-rate constrained communication channel. System (1) is equipped with a Sensor $\mathscr{S}$. The problem is depicted in Figure 1.

The sensor is responsible for generating the messages $m[k]$. In order to determine the content of each message, the sensor can use the set of all previously sent messages $M_{k}=\{m[j] \mid j$ : $k>j \geq 0\}$, the current output, as well as all previous output measurements. The sensor equation is then

$$
m[k]=\mathscr{S}\left(M_{k}, y_{1}[0], \ldots, y_{1}[k]\right),
$$

$\forall k \geq 0$. The messages are transmitted over a one-way data-rate constrained communication channel. The channel transports the messages from the sensor to the controller. For simplicity, we assume that there is no delay in the communication channel (i.e. message $m[k]$ is received at time $k$ by the decoder). The messages that the channel transports must belong to a finite-sized alphabet, which is a lists of symbols, from 1 to $l_{k}$, where $l_{k}$ is the cardinality of the alphabet at communication instant $k$, which is determined by an alphabet function $\mathscr{A}$ as follows

$$
l_{k}=\mathscr{A}\left(M_{k}\right),
$$

$\forall k \geq 0$. Since the messages should be part of the alphabet, we have the following restriction

$$
m[k] \in\left\{1, \ldots, l_{k}\right\},
$$

$\forall k \geq 0$. The length of each alphabet determines how many bits are necessary to encode the message. The number of bits required at each instant $b_{k}$ is defined as follows

$$
b_{k}:=\left\lceil\log _{2} l_{k}\right\rceil,
$$

$\forall k \geq 0$, where $\lceil\cdot\rceil$ is the ceiling function. The channel rate $c$ is the average number of bits that are sent per unit of time and is defined as follows

$$
c:=\operatorname{supinf}_{j \geq 0} \sum_{l \geq 1}^{l-1} \frac{b_{j+p}}{l} .
$$

On the other side of the communication channel, a controller $\mathscr{U}$ interprets the messages that it received to generate control inputs as follows

$$
u[k]=\mathscr{U}(m[0], \ldots, m[k]),
$$

$\forall k \geq 0$. We will design a communication protocol, in the form of a sensor (3), alphabet (4), and controller (8) that keeps the states of both systems within a certain distance from each other. To mathematically define this objective, we will need a quantity called the synchronization error which is defined as follows.

Definition 1: The synchronization error is $\eta:=$ $\sup _{k \geq 0}\left\|x_{1}[k]-x_{2}[k]\right\|_{2}$.

The design of the communication protocol should ensure that the synchronization error is bounded and that it requires a finite channel rate (as defined in (7)). In the following sections, we will design the communication protocol and investigate the relation between $\eta$ and $c$ for that particular communication protocol.

\section{The Communication Protocol}

Now that the problem has been stated, we describe the different components of the communication protocol. In order to minimize the distance between $x_{1}[k]$ and $x_{2}[k]$, the controller should apply a control input to system (2). The controller will thus need information about $x_{1}[k]$. The evolution over time of $x_{1}[k]$ is dictated by the dynamics of system (1) which can be separated in three categories: the linear mapping due to the matrix $A$, the reference signal $B r[k]$ 
and the perturbations $d_{i}[k]$. The first is known a priori but the latter two are not known as they are random.

In this section, we will first describe how we send messages with information about $x_{1}[k]$ and then we will describe the actual sensor, alphabet, and controller functions.

\section{A. What Types of Messages the Sensor Sends}

The sensor is responsible for sending messages with information about the reference signal and the perturbations. There are two difficulties related with transmitting information about $r[k]$ and $d_{1}[k]$ : firstly, the perturbations $d_{1}[k]$ are not measured, which implies that they cannot be sent in real time. Secondly, the channel uses finite-size alphabets, which can only encode sets of finite cardinality. Since the reference signal and perturbations are in $R \subset \mathbb{R}^{n_{r}}$ and $\mathbb{R}^{n_{x}}$ respectively, sending exact values of $r[k]$ and $d_{1}[k]$ is not possible as the latter would require an infinite number of bits. As a solution to these difficulties, we will send estimates $\hat{x}_{1}[k]$ and $\hat{r}[k]$, of $x_{1}[k]$ and $r[k]$ which will be part of sets with finite cardinalities.

The decision to transmit the estimates $\hat{r}[k]$ and $\hat{x}_{1}[k]$ are based on the messages that are sent, the dynamics of the underlying states, as well as an initial estimate of the state of system (1), which we assume that the sensor and controller possess, as a part of the communication protocol. This leads to the following assumption.

Assumption 4: The sensor and controller have access to an initial estimate $\hat{x}_{10}$ which verifies

$$
\left\|\hat{x}_{10}-x_{10}\right\|_{2} \leq \varepsilon
$$

All messages $m[k]$ will contain an estimate of the reference signal $\hat{r}[k]$. At some time instants, the messages also contain information about $\hat{x}_{1}[k]$ in addition to information about $\hat{r}[k]$. The sensor and controller use an auxiliary variable $h[k]$ which is 1 if an estimate of the state is communicated at time $k$ and 0 otherwise. The messages will be designed such that the controller can determine whether $h[k]=0$ or $h[k]=1$ when it receives a message $m[k]$. The estimates $\hat{r}[k]$ are computed directly from $m[k]$ at each time instant. The estimates $\hat{x}_{1}[k]$ are computed either from the message $m[k]$ if $h[k]=1$ or as a solution from the following system

$$
\begin{aligned}
\hat{x}_{1}[k+1] & =A \hat{x}_{1}[k]+B \hat{r}[k] \\
\hat{x}_{1}\left[k_{c}\right] & =\hat{x}_{\mathrm{lc}}[k],
\end{aligned}
$$

where $k_{c}[k]$ is the last time instant where $h\left[k_{c}\right]=1$ and $\hat{x}_{\mathrm{lc}}$ is the estimate stemming from the last received message. We will denote $\mathscr{D}$ the decoder, which is a part of the controller which is responsible for translating the messages $m[k]$ into estimates $\hat{r}[k]$ and $\hat{x}_{1}[k]$. The decoder equation is thus defined as

$$
\left(\hat{r}[k], \hat{x}_{1}[k]\right)=\mathscr{D}(m[k])
$$

Since the sensor has access to the initial estimate, and it sends the messages, it also maintains local estimates $\hat{r}_{c}[k]=\hat{r}[k]$ and $\hat{x}_{1 c}[k]=\hat{x}_{1}[k]$, which will be used to communicate.

\section{B. How to Send Estimates of the Reference Signal}

To generate $\hat{r}[k]$, a minimal covering of $R$ with balls of radius $\varepsilon_{r}$ is built. The centers of these balls are points $v_{l} \in R$, each with an index $l$. We denote $V_{R}$ the set of all the centers of the balls in the covering. We thus have $v_{l} \in V_{R}$ and $R \subseteq$ $\bigcup_{l=1}^{\left|V_{R}\right|} B_{\varepsilon_{r}}\left(v_{l}\right)$, where $\left|V_{R}\right|$ is the cardinality of the set $V_{R}$ and $B_{\varepsilon_{r}}(x)$, the ball of radius $\varepsilon_{r}$, centered in $x$. This covering is a part of the communication protocol and is known by both the sensor and the controller.

At each time instant, the indexes of the balls in the covering form part of or the entire alphabet for communication (only a part of the alphabet if an estimate of the state is also given). By sending a message that contains the index of one of the balls, the sensor provides an estimate of the reference signal $\hat{r}[k]$ to the decoder of system (2) whose precision is entirely determined by the choice of $\varepsilon_{r}$, the size of the balls in the covering $V_{R}$ of $R$.

\section{How to Send Estimates of the State of the First System}

In order to communicate $\hat{x}_{1}[k]$, it is not possible to use the same technique as for the reference signal, because the statespace is not bounded. Moreover, the state of the system is measured by the sensor with an error $w_{i}[k]$. Instead, we will employ the following technique which is inspired from [13] and [11] to communicate. If, $\left\|y_{1}[k]-\hat{x}_{1}[k]\right\|_{2} \leq \varepsilon_{x}$ at a certain time instant $k$, (the output of system (1) is in a ball of radius $\varepsilon_{x}$, whose center is an estimate that the controller knows), then we have $\left\|x_{1}[k]-\hat{x}_{1}[k]\right\|_{2} \leq \varepsilon_{x}+\omega$. At each consecutive time instant $k+1, k+2, \ldots$, the distance between the estimate and the state changes, due to the matrix $A$, the difference between $B r[k]$ and $B \hat{r}[k]$ and the perturbation $d_{1}[k]$. In the next lemma, we provide a bound on the maximum distance between the state of system (1) and the estimate of the state of system (1) after $\bar{k}$ time-steps, provided that initially they are in a ball of radius $\varepsilon_{x}+\omega$. We denote $\sigma_{i}(M)$ the singular values of the matrix $M$ in non-increasing order (i.e. $\sigma_{1}(M) \geq$ $\sigma_{2}(M) \geq \ldots \sigma_{n}(M) \geq 0$ ).

Lemma 1: For any $\varepsilon_{x}>0, k \geq 0, x_{1}[k], y_{1}[k], \hat{x}_{1}[k] \in$ $\mathbb{R}^{n_{x}}, w_{1}[k] \in \mathbb{R}^{n_{x}}$ satisfying Assumption 1 and such that $y_{1}[k]=x_{1}[k]+w_{1}[k]$ and $\left\|y_{1}[k]-\hat{x}_{1}[k]\right\|_{2} \leq \varepsilon_{x}$. For any $\bar{k} \geq 1, r[k], \ldots, r[k+\bar{k}-1], \hat{r}[k], \ldots, \hat{r}[k+\bar{k}-1] \in R, \varepsilon_{r}>0$ such that $\|r[j]-\hat{r}[j]\|_{2} \leq \varepsilon_{r}, \forall j \in\{k, \ldots, k+\bar{k}-1\}$, and $d_{1}[k], \ldots, d_{1}[k+\bar{k}-1] \in \mathbb{R}^{n_{x}}$ satisfying Assumption 1 , the following holds

$$
\begin{aligned}
\left\|x_{1}[k+j]-\hat{x}_{1}[k+j]\right\|_{2} & \leq \max \left\{\sigma_{1}(A)^{(\bar{k}-1)}, 1\right\}\left(\varepsilon_{x}+\omega\right) \\
& +\sum_{l=1}^{\bar{k}-1} \sigma_{1}(A)^{(l-1)}\left[\sigma_{1}(B) \varepsilon_{r}+\delta\right],
\end{aligned}
$$

$\forall j \in\{0, \ldots, \bar{k}-1\}$, where $x_{1}[k+j]$ is the solution of (1) starting at that particular $x_{1}[k]$ with those particular $r[k], \ldots, r[k+$ $\bar{k}-1]$ and $d_{1}[k], \ldots, d_{1}[k+\bar{k}-1]$, and $\hat{x}_{1}[k+j]$ is the solution of (9) starting at that particular $\hat{x}_{1}[k]$ with those particular $\hat{r}[k], \ldots, \hat{r}[k+\bar{k}-1]$, and $\delta$ and $\omega$ are taken from Assumption 1 .

The proof of this lemma has been omitted for space reasons. 
For brevity, we will denote

$$
\begin{aligned}
& \xi_{1}(\bar{k}):= \\
& \max \left\{\sigma_{1}(A)^{(\bar{k}-1)}, 1\right\}\left(\varepsilon_{x}+\omega\right)+\sum_{l=1}^{k-1} \sigma_{1}^{(l-1)}(A)\left[\sigma_{1}(B) \varepsilon_{r}+\delta\right]
\end{aligned}
$$

If the sensor sends a message containing information to generate a new estimate $\hat{x}_{1}[k]$ every $\bar{k}$ time instants, the previous lemma provides a bound on the maximum error between $x_{1}[k]$ and $\hat{x}_{1}[k]$. A new estimate of the state will be communicated at most every $\bar{k}$ time instants. In some cases, it is possible to delay the communication more than $\bar{k}$ time instants, while retaining the same error bound on the observation error. Since the sensor knows $\hat{x}_{1 c}[k]=\hat{x}_{1}[k]$, it knows which estimate the controller currently has. Instead of communicating a new estimate at time $\kappa=k+\bar{k}$, with $k \geq 0$, the sensor verifies whether the following inequality holds

$$
\begin{array}{r}
\left\|y_{1}[\kappa]-A \hat{x}_{1 c}[\kappa-1]-B \hat{r}_{c}[k-1]\right\| \leq \frac{1}{\sigma_{1}(A)} \max \left\{\sigma_{1}(A)^{\bar{k}}, 1\right\}\left(\varepsilon_{x}+\omega\right) \\
+\sum_{l=1}^{\bar{k}} \sigma_{1}(A)^{(l-2)}\left(\sigma_{1}(B) \varepsilon_{r}+\delta\right)-\frac{\sigma_{1}(B) \varepsilon_{r}+\delta}{\sigma_{1}(A)}-\omega
\end{array}
$$

whose validity implies that on the step $\kappa+1$ the inequality $\left\|x_{1}[\kappa+1]-\hat{x}_{1}[\kappa+1]\right\|_{2} \leq \xi_{1}(\bar{k})$ is satisfied and the error $e_{1}$ stays in the limit of (11). Hence, if the condition holds, then the sensor sends a message containing $\hat{r}[\kappa]$ only, and delays the communication to a later time instant (which implies that $h[\kappa]=0$ ), at which point the sensor will verify if (13) holds again and repeat the procedure. The estimates $\hat{x}_{1 c}[k]=\hat{x}_{1}[k]$ are simply computed as the solutions of (9) for that time instant as well. If at a certain time instant (13) does not hold, this triggers a communication with a new estimate (which implies that $h[\kappa]=1$ ). Since there are at least $\bar{k}$ instants in between two consecutive communications with estimates of the state, Zeno-behavior is impossible.

We want to build a covering of the ball centered around $\hat{x}_{1}[k]$ of radius sufficiently large such that $y_{1}[k]$ is contained in the ball at times $k$ such that $h[k]=1$ (which implies that either exactly $\bar{k}$ time instants have passed since the last communication instants, or (13) was true at the previous time instant). The following lemma provides a bound on the radius of such a ball.

Lemma 2: Let there be $k \geq 1, \varepsilon_{x}>0, \varepsilon_{r}>0 \quad y_{1}[k-$ 1], $y_{1}[k], x_{1}[k-1], x_{1}[k], \hat{x}_{1}[k] \in \mathbb{R}^{n_{x}}, r[k-1] \in R, w_{1}[k], w_{1}[k-$ 1] $\in \mathbb{R}^{n_{x}}$ and $d_{1}[k] \in \mathbb{R}^{n_{x}}$ such that Assumption 1 holds and $y_{1}[k-1]=x_{1}[k-1]+w_{1}[k-1], x_{1}[k]=A x_{1}[k-1]+B r[k-$ $1]+d_{1}[k-1]$ and $y_{1}[k]=x_{1}[k]+w_{1}[k]$. Then both of the following conditions

$$
\begin{aligned}
\left\|x_{1}[k-1]-\hat{x}_{1}[k-1]\right\| & \leq \max \left\{\sigma(A)^{(\bar{k}-1)}, 1\right\}\left(\varepsilon_{x}+\omega\right) \\
& +\sum_{l=1}^{k-1} \sigma_{1}(A)^{(l-1)}\left[\sigma_{1}(B) \varepsilon_{r}+\delta\right],
\end{aligned}
$$

and

$$
\begin{array}{r}
\left\|y_{1}[k-1]-\hat{x}_{1}[k-1]\right\| \leq \frac{1}{\sigma_{1}(A)} \max \left\{\sigma_{1}(A)^{\bar{k}}, 1\right\}\left(\varepsilon_{x}+\omega\right) \\
+\sum_{l=1}^{\bar{k}} \sigma_{1}(A)^{(l-2)}\left(\sigma_{1}(B) \varepsilon_{r}+\delta\right)-\frac{\sigma_{1}(B) \varepsilon_{r}+\delta}{\sigma_{1}(A)}-\omega
\end{array}
$$

imply that

$$
\begin{aligned}
\left\|y_{1}[k]-\hat{x}_{1}[k]\right\| & \leq \max \left\{\sigma(A)^{\bar{k}}, 1\right\}\left(\varepsilon_{x}+\omega\right)+\omega \\
& +\sum_{l=1}^{\bar{k}} \sigma_{1}(A)^{(l-1)}\left[\sigma_{1}(B) \varepsilon_{r}+\delta\right],
\end{aligned}
$$

where $\omega$ and $\delta$ are taken from Assumption 1 .

The proof of this lemma has been omitted for space reasons. For brevity, we will use the following notation for the bound obtained in the previous lemma

$$
\begin{aligned}
& \xi_{2}(\bar{k}):= \\
& \max \left\{\sigma(A)^{\bar{k}}, 1\right\}\left(\varepsilon_{x}+\omega\right)+\omega+\sum_{l=1}^{\bar{k}} \sigma_{1}(A)^{l-1}\left[\sigma_{1}(B) \varepsilon_{r}+\delta\right]
\end{aligned}
$$

In order to send a message containing a new estimate of precision $\varepsilon_{x}$ at $k$ (where $h[k]=1$ ), the sensor starts by computing the set containing $y_{1}[k]$ centered around $\hat{x}_{1}[k]$ at the time of communication. If this set is a ball of radius $\xi_{2}(\bar{k})$, it depends on parameters that are known by both the sensor and controller, which implies that both the sensor and controller can build the set (because they know $\hat{x}_{1 c}[k]=\hat{x}_{1}[k]$ ). By covering the set with balls of radius $\varepsilon_{x}$, and indexing the balls, we again have an alphabet for communication. Because the covering is done with balls of radius $\varepsilon_{x}$, at the times of communications, we would have $\left\|y_{1}[k]-\hat{x}_{1}[k]\right\|_{2} \leq \varepsilon_{x}$ and hence $\left\|x_{1}[k]-\hat{x}_{1}[k]\right\|_{2} \leq \varepsilon_{x}+\omega$, which implies that Lemma 1 can be used again to provide a bound on the distance between $x_{1}[k]$ and $\hat{x}_{1}[k]$ until the next communication instant (at least $\bar{k}$ time instants later).

A covering of $B_{\xi_{2}(\bar{k})}\left(\hat{x}_{1}[k]\right)$ with balls of radius $\varepsilon_{x}$ is built at times $k$ such that $h[k]=1$. The centers of these balls are points $v_{j}^{k} \in B_{\xi_{2}(\bar{k})}\left(\hat{x}_{1}[k]\right)$, each with an index $j$. We will call $V_{x}^{k}$ the set of all the centers of the balls in the covering at time $k$. We thus have $v_{j}^{k} \in V_{x}^{k}$ and $R \subseteq \bigcup_{j=1}^{\left|V_{x}^{k}\right|} B_{\varepsilon_{x}}\left(v_{j}^{k}\right)$. This covering is a part of the communication protocol and is known by both the sensor and the controller. Since all balls $B_{\xi_{2}(\bar{k})}\left(\hat{x}_{1}[k]\right)$ have the same radius, building a covering of them is a computationally easy task: it suffices to build a covering of $B_{\xi_{2}(\bar{k})}(0)$ and shift this covering by $\hat{x}_{1}[k]$ at the times $k$ where $h[k]=1$.

Remark 2: Note that to reconstruct the current estimate, only the estimate stemming from the last message and the new message are required. There is no need to store all previously sent messages which implies that the need for local storage capacity is very limited.

Before we present the final equations of the consensus protocol, we will need some notations. At each time $k, l_{k}$ is the index of the center of the ball of radius $\varepsilon_{r}$ in the covering 


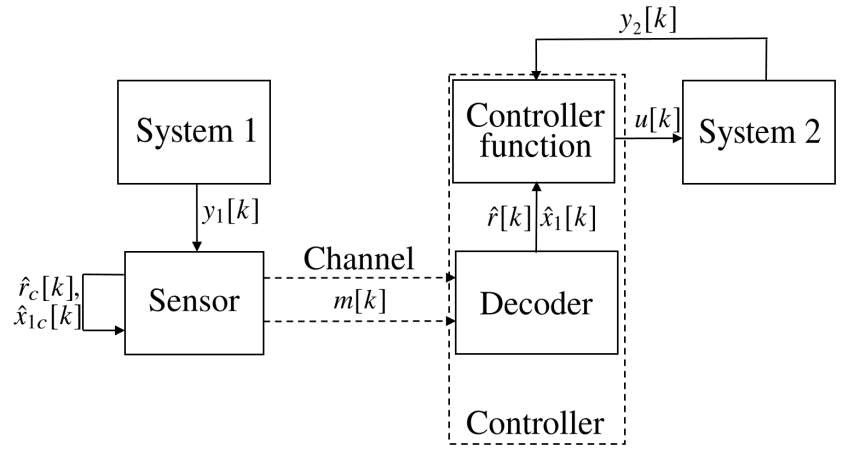

Fig. 2. Structure of the setup.

$V_{R}$ closest to the reference signal $r[k]$. At each time $k$ such that $h[k]=1, j_{k}$ is the index of the ball of radius $\varepsilon_{x}$ in the covering $V_{x}^{k}$ closest to the output $y_{1}[k]$.

\section{The Communication Protocol Functions}

We now have the necessary ingredients to define the sensor function $\mathscr{S}$, alphabets function $\mathscr{A}$, decoder $\mathscr{D}$ and controller $\mathscr{U}$. For clarity, the different components of the communication protocol are depicted in Figure 2. For brevity, we do not repeat the arguments of each functions and use the notation $\mathscr{S}(\cdot), \mathscr{A}(\cdot), \mathscr{D}(\cdot), \mathscr{U}(\cdot)$ instead. The sensor function (3) is

$$
\mathscr{S}(\cdot)=\left\{\begin{array}{cl}
l_{k} & \forall k: h[k]=0, \\
l_{k} \stackrel{\sim}{\sim} j_{k} & \forall k: h[k]=1,
\end{array}\right.
$$

where $A \frown B$ refers to the concatenation of two messages (i.e. "12" " $34 "=$ "1234"). The alphabet function (4) is

$$
\mathscr{A}(\cdot)=\left\{\begin{array}{cc}
\left|V_{R}\right|, & \forall k: h[k]=0, \\
\left|V_{R}\right|\left|V_{x}^{k}\right|, & \forall k: h[k]=1 .
\end{array}\right.
$$

The decoder function (10) is

$$
\mathscr{D}(\cdot)=\left\{\begin{array}{cc}
\left(v_{l_{k}}, A \hat{x}_{1}[k-1]+B \hat{r}[k-1]\right), & \forall k: h[k]=0, \\
\left(v_{l_{k}}, v_{j_{k}}\right), & \forall k: h[k]=1 .
\end{array}\right.
$$

The controller function (8) is

$$
\mathscr{U}(\cdot)=\hat{r}[k]-K \hat{x}_{1}[k]+K y_{2}[k] .
$$

where $K$ is the controller gain. Note that a linear controller was used here for simplicity. It is possible to replace it by a more sophisticated controller to improve the performance of the communication protocol. However, due to page number limitations, we do not explore this option in this paper.

The communication protocol (19), (20), (21), and (22) doesn't guarantee that the synchronization error $\eta$ will be finite. For some values of $A, B, R, \delta$ and $\omega$, and for some choices of $\bar{k}, \varepsilon_{r}, \varepsilon_{x}$, and $K$ this might be the case, but for others certainly not. In the next section, we will address the question of the choice of $\bar{k}, \varepsilon_{r}, \varepsilon_{x}$ and their influence on $\eta$ and $c$.

\section{BOUNDS ON THE SYNCHRONIZATION ERROR AND CHANNEL RATE}

In this section, we provide two results: firstly, we compute the control gain $K$ as a nonlinear program and estimate a bound on $\eta$ with that choice of $K$. Secondly, we provide a bound on $c$ for the communication protocol that was defined in the previous section.

The controller gain is computed as a solution of the following nonlinear program:

$$
\begin{array}{r}
\left(K, \gamma_{1}^{*}\right)=\arg \min _{K \in \mathbb{R}^{n_{r} \times n_{x}, \gamma_{1} \in \mathbb{R}^{+}}} \sigma_{1}(K)\left(\xi_{1}(\bar{k})+\omega\right)-\gamma_{1} \\
\text { s.t. } \sigma_{1}(A+B K)<1-\gamma_{1},
\end{array}
$$

Remark 3: The program (23) can be reformulated as an LMI. For brevity, the LMI reformulation is not presented in this document. This program is always feasible if there exists $K$ such that $\sigma_{1}(A+B K)<1$, which is an inequality that requires a property similar to the stabilizability of the pair $(A, B)$. It is not the same as stabilizability since there exist stabilizable systems for which no $K$ that guarantees $\sigma_{1}(A+$ $B K)<1$ exists, in that sense the condition $\sigma_{1}(A+B K)<1$ is stricter than the stabilizability of $(A, B)$. It is possible to adapt the results of this paper to only assume stabilizability but such details are left for further research.

If the previous program has a solution, the boundedness of the synchronization error $\eta$ is ensured, as it is proved in the next theorem.

Theorem 1: Let Assumptions 1 to 4 hold for the systems (1) and (2). Assume that (23) has a solution. Then for any choices of $\varepsilon_{r}>0$ and $\varepsilon_{x}>0$, the consensus protocol (19), (20) and (22) with a choice of $K$ as in (23) leads to the following inequality

$$
\eta \leq \frac{\sigma_{1}(B) \varepsilon_{r}+2 \delta+\sigma_{1}(B K)\left(\xi_{1}(\bar{k})+\omega\right)}{1-\sigma_{1}(A+B K)}, \quad \forall k \geq 0,
$$

where $\xi_{1}(\bar{k})$ is defined in (12).

The proof of this theorem has been omitted for space reasons.

The next theorem provides a bound on the channel rate $c$, in function of the different parameters of the problem. For this theorem, we will need the diameter $\rho_{R}$ of the set $R$, which is defined as follows $\rho_{R}:=\max _{r_{1}, r_{2} \in R}\left\|r_{1}-r_{2}\right\|_{2}$.

Theorem 2: Let Assumptions 1 to 4 hold for the systems (1) and (2). Assume (23) has a solution. Then for any choices of $\varepsilon_{r}>0$ and $\varepsilon_{x}>0$, the consensus protocol (19), (20), and (22), leads to

$$
c \leq\left\lceil n_{r} \log _{2}\left\lceil\frac{\sqrt{n_{r}} \rho_{R}}{\varepsilon_{r}}\right\rceil\right\rceil+\frac{1}{\bar{k}}\left\lceil n_{x} \log _{2}\left\lceil\frac{\sqrt{n_{x}} \xi_{2}(\bar{k})}{\varepsilon_{x}}\right\rceil\right\rceil,
$$

where $\xi_{2}(\bar{k})$ is defined in (18).

The proof of this theorem has been omitted for space reasons.

Remark 4: Theorems 1 and 2 provide tools to choose $\bar{k}$, $\varepsilon_{r}$, and $\varepsilon_{x}$. The larger $\bar{k}$ is chosen, the smaller the required channel rate is and the larger the error is. The smaller $\varepsilon_{r}$ and $\varepsilon_{x}$ are, the smaller the error is, but also the larger the required channel rate is. It is thus possible to exchange accuracy for a higher rate and vice versa. 


\section{Simulations}

In this section, we apply the previously designed communication protocol. We are going to apply Theorems 1 and 2 to compute the bounds on $\eta$ and $c$. We will then compare the actual rate required by the communication protocol.

We consider the systems (1) and (2) with

$$
A=\left[\begin{array}{cc}
1.2505 & 0.6236 \\
-0.1525 & 0.4837
\end{array}\right], B=\left[\begin{array}{c}
-0.0524 \\
0.2188
\end{array}\right]
$$

the set $R=B_{10}(0), \delta=1$, and $\omega=0.1$. Note that systems are unstable since $\lambda_{1}(A)=1.0949$ (where $\lambda_{i}(A)$ are the eigenvalues of the matrix $A$, in non-increasing order). We choose $\varepsilon=10^{-2}, \varepsilon_{x}=1, \varepsilon_{r}=1$. The controller gain was computed via (23) to be $K=[2.63062 .8906]$. We tested the communication protocol for several choices of $\bar{k}$ by MonteCarlo simulations with 100,000 iterations each from $k=0$ till $k=100$ with $(0,0)$ as initial conditions for both systems and with randomly generated driving signals $r[k] \in[-10,10]$. The resulting error bounds $\eta$, theoretical rates $c$, and actual rates $c^{*}$ are displayed in Table I. In addition, we display $c-c_{r}$, and $c^{*}-c_{r}$, where $c_{r}$ is the rate of the channel necessary to transmit $r$. Figure 3 shows the observation error $\left\|x_{1}[k]-\hat{x}_{1}[k]\right\|_{2}$ as well as the difference between the states of both systems $\left\|x_{1}[k]-x_{2}[k]\right\|_{2}$ for one of the simulations (with $\bar{k}=5)$. The green line is the right-hand side of $(13)$, which is the bound that the sensor verifies in order to determine if an estimate of the state is sent, and the red line is $\eta$, the maximum allowable synchronization error.

We make the following observations. By increasing $\bar{k}$ (see Table I), the error increases. The theoretical rate $c$ is reduced by an increase in $\bar{k}$, as was expected. The actual rate is below the theoretical values (sometimes half of the theoretical value), so the fact that the sensor functions in an event-triggered manner clearly improves the rate. The actual effect of the scheme is much clearer when we compare $c-c_{r}$ and $c^{*}-c_{r}$ as that is the part of the rate that is affected by the event-triggering mechanism. For that part of the rate, the theoretical rate is sometimes ten times larger than the simulated rate. This effect is reduced for large values of $\bar{k}$.

Regarding Figure 3, we can see that the observation error indeed resets every time it approaches the bound that is imposed on the sensor. We can see that we indeed communicate less often than every five time instants. After each communication, the observation error is reset to $\varepsilon_{x}$ and the synchronization error follows at the next time instant. The error $\left\|x_{1}[k]-x_{2}[k]\right\|_{2}$ stays well below $\eta$, which is due to the conservatism of the bounds. This conservatism could be reduced by using different norms, as well as more tightly defining the region enclosed by (13).

\section{REFERENCES}

[1] B. Andrievsky, A. Matveev, and A. Fradkov. Control and Estimation under Information Constraints: Toward a Unified Theory of Control, Computation and Communications. Automation and Remote Control, 71:572-633, 2010.

[2] J. Baillieul and P. J. Antsaklis. Control and Communication Challenges in Networked Real-Time Systems. Proceedings of the IEEE, 95(1):928, 2007.

\begin{tabular}{|c|c|c|c|c|c|}
\hline $\bar{k}$ & 2 & 3 & 5 & 10 & 20 \\
\hline$\eta$ & 13.0697 & 20.3852 & 41.4596 & 231.846 & 6616.53 \\
\hline$c$ & 10 & 9 & 7.8 & 7 & 6.45 \\
\hline$c^{*}$ & 5.55725 & 5.46537 & 5.35751 & 5.27247 & 5.23249 \\
\hline$c-c_{r}$ & 5 & 4 & 2.8 & 2 & 1.45 \\
\hline$c^{*}-c_{r}$ & 0.55725 & 0.46537 & 0.35751 & 0.27247 & 0.23249 \\
\hline
\end{tabular}

TABLE I

RESULTS FOR VARIOUS $\bar{k}$

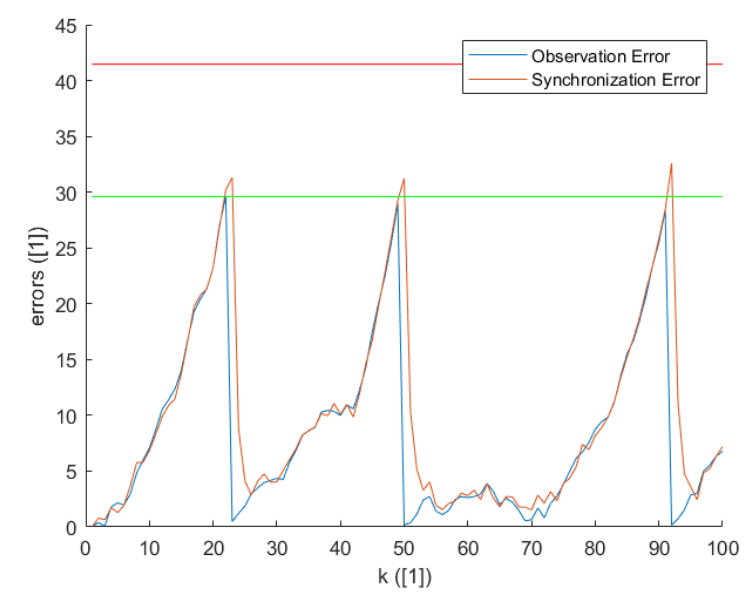

Fig. 3. Evolution over time of the errors for one simulation with $\bar{k}=5$.

[3] W. Dong. Consensus of High-Order Nonlinear Continuous-Time Systems With Uncertainty and Limited Communication Data Rate. IEEE Transactions on Automatic Control, 64(5):2100-2107, 2019.

[4] N. Elia and S. Mitter. Stabilization of linear systems with limited information. IEEE Transactions on Automatic Control, 46(9):13841400,2001

[5] A. L. Fradkov, B. Andrievsky, and R. J. Evans. Controlled synchronization under information constraints. Physical Review E - Statistical, Nonlinear, and Soft Matter Physics, 78(3):1-6, 2008.

[6] A. L. Fradkov, B. Andrievsky, and R. J. Evans. Synchronization of nonlinear systems under information constraints. Chaos, 18(3):037109-8, 2008.

[7] H. Li, G. Chen, T. Huang, Z. Dong, W. Zhu, and L. Gao. EventTriggered Distributed Average Consensus over Directed Digital Networks with Limited Communication Bandwidth. IEEE Transactions on Cybernetics, 46(12):3098-3110, 2016.

[8] T. Li, M. Fu, L. Xie, and J.-f. Zhang. Distributed Consensus With Limited Communication Data Rate. IEEE Transactions on Automatic Control, 56(2):279-292, 2011.

[9] T. Li and L. Xie. Distributed consensus over digital networks with limited bandwidth and time-varying topologies. Automatica, 47(9):2006-2015, 2011.

[10] G. Nair, F. Fagnani, S. Zampieri, and R. Evans. Feedback Control Under Data Rate Constraints: An Overview. Proceedings of the IEEE, 95(1):108-137, 2007.

[11] Q. Voortman, D. Efimov, A. Pogromsky, J. P. Richard, and H. Nijmeijer. Event-triggered Data-efficient Observers of Perturbed Systems. In Accepted for publication at the IFAC World Congress, 2020.

[12] Q. Voortman, A. Pogromsky, A. Matveev, and H. Nijmeijer. Consensus of Nonlinear Systems with Data-Rate Constraints. Accepted for publication at the IFAC World Congress, 2020.

[13] Q. Voortman, A. Y. Pogromsky, A. S. Matveev, and H. Nijmeijer. Data-Rate Constrained Observers of Nonlinear Systems. Entropy, 21(282):1-29, 2019.

[14] W. S. Wong and R. W. Brockett. Systems with finite communication bandwidth constraints - Part I: State estimation problems. IEEE Transactions on Automatic Control, 42(9):1294-1299, 1997. 